\title{
Are there attacking points in the eicosanoid cascade for chemotherapeutic options in benign meningiomas?
}

\author{
Christina Pfister, B.Sc., ${ }^{1}$ Rainer Ritz, M.D. ${ }^{1}$ Heike Pfrommer, ${ }^{1}$ \\ Antje Bornemann, M.D., Ph.D., ${ }^{2}$ Marcos S. Tatagiba, M.D., Ph.D., ${ }^{1}$ \\ AND Florian Roser, M.D., PH.D. ${ }^{1}$ \\ ${ }^{1}$ Department of Neurosurgery and the ${ }^{2}$ Institute of Brain Research, University of Tübingen, Germany
}

\begin{abstract}
Object. The current treatment for recurrent or malignant meningiomas with adjuvant therapies has not been satisfactory, and there is an intense interest in evaluating new molecular markers to act as therapeutic targets. Enzymes of the arachidonic acid (AA) cascade such as cyclooxygenase (COX)-2 or 5-lipoxygenase (5-LO) are upregulated in a number of epithelial tumors, but to date there are hardly any data about the expression of these markers in meningiomas. To find possible targets for chemotherapeutic intervention, the authors evaluated the expression of AA derivatives at different molecular levels in meningiomas.

Methods. One hundred and twenty-four meningioma surgical specimens and normal human cortical tissue samples were immunohistochemically and cytochemically stained for COX-2, COX-1, 5-LO, and prostaglandin E receptor 4 (PTGER4). In addition, Western blot and polymerase chain reaction (PCR) analyses were performed to detect the presence of eicosanoids in vivo and in vitro.

Results. Sixty (63\%) of 95 benign meningiomas, 21 (88\%) of 24 atypical meningiomas, all five malignant meningiomas, and all normal human cortex samples displayed high COX-2 immunoreactivity. All cultured specimens and IOMM-Lee cells stained positive for COX-2, COX-1, 5-LO, and PTGER4. The PCR analysis demonstrated no changes in eicosanoid expression among meningiomas of different World Health Organization grades and in normal human cortical and dura mater tissue.

Conclusions. Eicosanoid derivatives COX-1, COX-2, 5-LO, and PTGER4 enzymes show a high universal expression in meningiomas but are not upregulated in normal human cortex and dura tissue. This finding of the ubiquitous presence of these enzymes in meningiomas offers an excellent baseline for testing upcoming chemotherapeutic treatments. (DOI: $10.3171 /$ FOC-07/10/E8)
\end{abstract}

\section{KEY WORDS • chemotherapy • eicosanoid • meningioma • polymerase chain reaction - Western blot}

$\mathrm{M}$

ENINGIOMAS are usually benign tumors that arise from the leptomeningeal cells of the arachnoid membrane surrounding the brain and spinal cord. However, recurrence after seemingly complete surgical removal occurs in eight to $15 \%$ of cases, and tumor control rates achieved with radiotherapy are only $80 \% .^{20,35,36,53}$ Moreover, a surgical cure is not always achievable in meningiomas involving the skull base or vital neurovascular structures. Results achieved with chemotherapeutic treatments in the past were not convincing, and even drugs such as temozolomide-which have shown high efficacy

Abbreviations used in this paper: $\mathrm{AA}=$ arachidonic acid; $\mathrm{COX}=$ cyclooxygenase; LO = lipoxygenase; PBS = phosphate-buffered saline; $\mathrm{PCR}=$ polymerase chain reaction; $\mathrm{PG}=$ prostaglandin; PTGER4 = prostaglandin $\mathrm{E}$ receptor $4 ; \mathrm{WHO}=$ World Health Organization. against malignant brain tumors - have failed to inhibit the growth of refractory meningiomas in Phase II studies. ${ }^{7,26,39,45}$ The development of novel treatment strategies based on molecular information has not yet been successfully translated into common clinical practice.

Arachidonic acid is a v6 polyunsaturated fatty acid that is converted into biologically active lipid compounds called eicosanoids. Eicosanoids constitute a large family of biologically active lipid mediators produced by two enzyme classes: the cyclooxygenases (COX-1 and COX-2) and the lipoxygenases (5-LO, 12-LO, and 15-LO). Both classes catalyze the same enzymatic reaction that occurs in the synthesis of $\mathrm{PG}_{2}$ and $\mathrm{PGH}_{2}$, which are successively metabolized to $\mathrm{PGE}_{2}, \mathrm{PGD}_{2}, \mathrm{PGF}_{2}$, thromboxane $\mathrm{A}_{2}$, and prostacyclin $\mathrm{PGI}_{2}$ (Fig. 1). ${ }^{12}$

The crucial role of COX enzymes has been clearly demonstrated in human colon cancer cells, in which COX2 is often overexpressed. ${ }^{30,40}$ Cyclooxygenase inhibitors, 


\section{Pfister et al.}

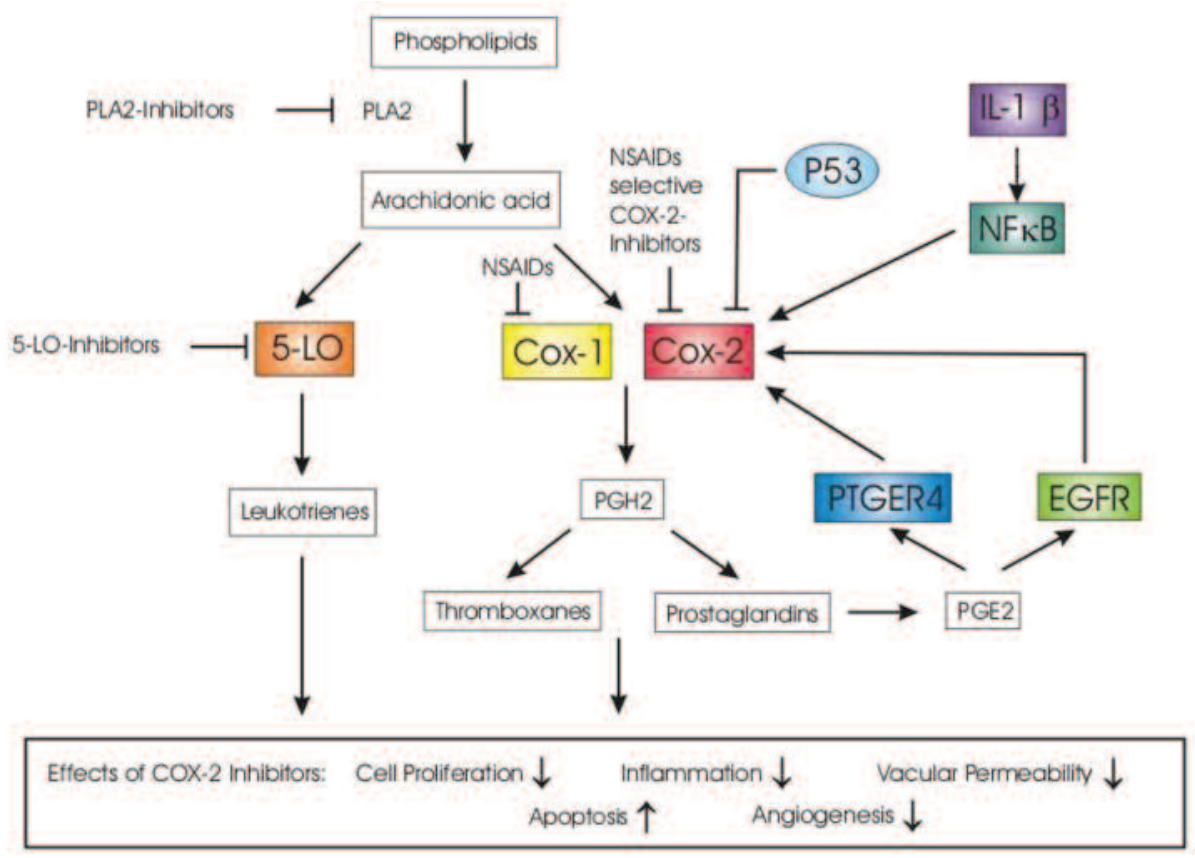

FIG. 1. Simplified diagram demonstrating the AA cascade and the enzymes produced. EGFR = epidermal growth factor receptor; $\mathrm{IL}=$ interleukin; $\mathrm{NF \kappa} B=$ nuclear factor- $\kappa \mathrm{B} ; \mathrm{NSAIDs}=$ nonsteroidal antiinflammatory drugs; PLA2 = phospholipase A2.

such as nonsteroidal antiinflammatory drugs and COX2-specific inhibitors, can reduce the incidence of colorectal cancer in humans and experimental animal models, and can decrease the number and size of polyps in patients with familial adenomatous polyposis. ${ }^{13}$ Recently, gliomas and meningiomas were also found to overexpress both $\mathrm{COX}$ and LO enzymes compared with normal brain tissue. , $^{6,37}$ Matsuo and coworkers ${ }^{32}$ reported that the staining intensity in glioblastomas was relatively weak, but that meningiomas and WHO Grade II/III astrocytomas were strongly positive for COX-2. Although much attention has been focused on the role of $\mathrm{COX}$-derived metabolites in cancer development and progression, accumulating evidence suggests that 5-LO-derived eicosanoids may play an equally important role. 5-lipoxygenase mRNA and/or protein was found to be overexpressed in human breast, pancreatic, or colon cancer cells. ${ }^{17,21,49}$ Boado et al. ${ }^{3}$ reported increased expression of 5-LO mRNA in three of three meningioma surgical specimens examined.

Most tumors that express COX have been found to contain high levels of $\mathrm{PGE}_{2}{ }^{10}$ Presumably, this especially bioactive lipid product of COX is responsible for some of the proneoplastic effects mediated by the enzymes such as inducing growth, migration, and invasiveness in colorectal carcinoma cells. ${ }^{5}$ Little is known of the production of PGs in intracranial tumors; some authors have reported elevated $\mathrm{PGE}_{2}$ levels in brain tumors in general and significantly higher $\mathrm{PGE}_{2}$ level in meningiomas. ${ }^{9,25}$ In contrast, other authors have reported no increase brain tumors' production of $\mathrm{PGE}_{2}{ }^{14}$ In the present study, we demonstrate the ubiquitous expression of AA derivatives in human meningiomas with immunohistochemical staining, Western blot analysis, and PCR.

\section{Materials and Methods}

\section{Tumor Specimens and Cell Culture}

Meningioma surgical specimens were obtained from the Neurosurgical Department in accordance with regulations of the Ethics Committee of the University of Tübingen. Primary cultures were obtained from tumor tissue samples within 15 minutes of surgical removal. The samples were first washed in PBS, reduced and mashed through a filter and placed in Dulbecco modified Eagle's medium with fetal bovine serum, $2 \mathrm{mmol} / \mathrm{L}$ L-glutamine, and $0.1 \% 10$ $\mathrm{mg} / \mathrm{ml}$ gentamicin (Invitrogen). The cells were plated in $12.5-\mathrm{mm}^{2}$ tissue culture flasks and incubated at $37^{\circ} \mathrm{C}$ in a humidified atmosphere of $5 \% \quad \mathrm{CO}_{2}$. The medium was changed every 3 to 4 days, and cultures were split using $300-\mu 1$ accutase (PAA). Viable cells were frozen in liquid nitrogen in $90 \%$ medium $/ 10 \%$ dimethyl sulfoxide. As there is no $\mathrm{PGE}_{2}$ antibody commercially available, the subtype 4 of the prostaglandin receptor E2 was selected for the studies because it is the only prostaglandin E2 receptor subtype that is expressed in brain. The IOMM-Lee cell line was a kind gift from Dr. Anita Lal (Department of Neurological Surgery, University of California, San Francisco).

\section{Immunohistochemical Staining}

Four-micron sections containing human meningiomas were cut from formalin-fixed tissue embedded in paraffin blocks. Normal cerebral cortex (Biochain Inc.), colon, and tonsil tissues were used as positive controls. Slides containing tissue were deparaffinized by bathing them in a series of histolene (Engelbrecht) and alcohol solutions. Vectastain Elite Universal Kits (Vector Laboratories) were used according to the manufacturer's protocol. Briefly, the 
slides were treated with an antigen unmasking solution (citrate buffer solution at $\mathrm{pH}$ 6.0), rinsed with PBS, and bathed in a $3 \% \mathrm{H}_{2} \mathrm{O}_{2}$ solution for 10 minutes, followed by blocking with avidinbiotin (Vector Laboratories). Slides were incubated overnight at $4^{\circ} \mathrm{C}$ with primary COX-2 mouse monoclonal antibody (160112, Cayman Chemical) at a dilution of 1:50, COX-1 mouse monoclonal antibody (160110, Cayman Chemical) at a dilution of 1:100, 5-LO rabbit polyclonal antibody (101775, Cayman Chemical) at a dilution of 1:100, and PTGER4 rabbit polyclonal antibody (160402, Cayman Chemical) at a dilution of 1:50. After the samples were incubated with the primary antibody and washed twice with PBS, biotinylated secondary antibody was applied, and the samples were incubated with premixed avidin-biotin peroxide complex (Vector Laboratories). The final immunohistochemical staining was performed using Vector NovaRED (Vector Laboratories) to obtain a reaction to the avidin-biotin complex. Sections were counterstained with hematoxylin and examined under light microscopy.

The immunohistochemical samples were graded using a five-point scoring system to describe the percentage of cells that demonstrated positive staining, as previously published. ${ }^{44}$ The scores were as follows: 0, no staining noted; 1 , less than $1 \%$ of cells stained; 2,1 to $10 \%$ of cells stained; 3,11 to $50 \%$ of cells stained; and 4, more than $50 \%$ of cells stained.

\section{Immunocytochemical Staining}

Immunocytochemical analysis was performed on meningioma cells growing in a monolayer fashion in culture. The cells were subjected to treatment, plated in four-well glass slides, and allowed to remain in growth media for 2 to 3 days as described in the previous section. After removal of the growth media, the slides were rinsed with PBS for 5 minutes. Methanol maintained at $-20^{\circ} \mathrm{C}$ was added to each well for 10 minutes. After the methanol had been removed, the slides were rinsed twice with PBS and bathed in 3\% $\mathrm{H}_{2} \mathrm{O}_{2}$ solution for 5 minutes to quench endogenous peroxidases. The incubation and staining protocols are identical to the immunohistochemical stainings described earlier.

\section{Protein and RNA Isolation}

Cytoplasmic and nuclear protein and RNA were isolated from surgical specimens and primary cell cultures using the protein and RNA isolation system (PARIS, Ambion, Inc.) according to the manufacturer's protocol. The nuclear and cytoplasmic protein and RNA was measured spectrophotometrically. The probes were stored at $-80^{\circ} \mathrm{C}$ until use.

\section{Reverse Transcription and PCR}

Ribonucleic acid samples were treated with DNase using the DNAfree kit (Ambion, Inc.) prior to reverse transcription-PCR analysis. A $0.4-\mu \mathrm{g}$ sample of cytoplasmic and nuclear RNA was reverse-transcribed using random hexamer primers and the Transcriptor First Strand cDNA Synthesis kit (Roche) according to the manufacturer's protocol. The PCR was performed with $50 \mathrm{ng}$ of cDNA in a $50-\mu l$ reaction mixture containing FastStart Taq DNA Polymerase dNTPack (Roche) according to the manufacturer's protocol, with COX-2, COX-1, ACTB (loading control), PTGER4 primer (SuperArray), and 5-LO primer: forward, 5'-TCTCAAGCAACACCGACGTAAAG-3'; reverse, 5'-TGCATGAAGCGGTTGATGAA-3' designed based on the sequence of human 5-LO cDNA. ${ }^{40}$ The PCR conditions for COX-2, COX-1, ACTB, and PTGER4 were $95^{\circ} \mathrm{C}$ for 15 minutes, 40 cycles of $95^{\circ} \mathrm{C}$ for 30 seconds, $55^{\circ} \mathrm{C}$ for 30 seconds and $72^{\circ} \mathrm{C}$ for 30 seconds, and one cycle of $72^{\circ} \mathrm{C}$ for 10 minutes. For 5-LO the conditions were 40 cycles at $95^{\circ} \mathrm{C}$ for 45 seconds, $60^{\circ} \mathrm{C}$ for 1 minute, $72^{\circ} \mathrm{C}$ for 1 minute, and one cycle at $72^{\circ} \mathrm{C}$ for 10 minutes. Polymerase chain reaction products $(16 \mu \mathrm{l})$ were subjected to electrophoresis on $10 \%$ TBE-Gel, and stained with the SilverQuest Silver Staining Kit (LC6070, Invitrogen, Inc.). The intensity of expression for each reaction was analyzed using a semiquantitative grading system as follows: Grade 0 , no expression; Grade 1, minimal expression; Grade 2, moderate expression; Grade 3, marked expression; and Grade 4, strong expression.

\section{Western Blot Analysis}

Equal amounts of protein $(\sim 100 \mu \mathrm{g})$ were resolved using sodium dodecyl sulfate-polyacrylamide gel electrophoresis (Nupage Bis-Tris-Gel 4-12\%, Invitrogen, Inc.) and transferred to polyvinylidene difluoride Hybond-P membrane (Invitrogen, Inc.). The membrane was blocked and probed using a COX-2 mouse monoclonal antibody (160112, Cayman Chemical) at a dilution of 1:1000, COX1 mouse monoclonal antibody (160110, Cayman Chemical) at a dilution of 1:100,5-LO rabbit polyclonal antibody (101775, Cayman Chemical) at a dilution of 1:250, and PTGER4 rabbit polyclonal antibody (160402, Cayman Chemical) at a dilution of 1:200. An antibody to $\beta$-actin (1:1000; Ab8226, Abcam) was used to confirm equal loading among the samples. The Western blots were visualized using the Western Breeze Chromogenic Kit (Invitrogen, Inc.). The intensity of expression for each reaction was analyzed using the same semiquantitative grading system already described.

\section{Statistical Analysis}

Results are expressed as the means \pm the standard error of the means. Statistical analysis was performed using the unpaired t-test. Probability values less than 0.05 were considered statistically significant.

\section{Results}

\section{Patient Demographics and Tumor Characteristics}

Tumor samples were obtained in 124 patients. The patients ranged from 22 to 88 years of age (mean 61 years), and $98(79 \%)$ were women. Although 95 tumors (77\%) were histologically benign, there were 24 atypical (19\%) and five anaplastic meningiomas (4\%). For the in vitro immunohistochemical staining studies, seven primary cell cultures were grown in monolayer cultures. Seventy-one percent of the cell lines had been obtained in female patients; the cell lines were derived from two benign, one atypical, one chordoid meningioma, and two malignant (IOMM-Lee) meningiomas. The primary cell cultures were immunohistochemically stained with epithelial membrane antigen and vimentin antibody to verify that they were 
meningioma cells. A strong correlation between the Ki 67 proliferation index and the growth of tumor cells in culture has been seen ( $p<0.001$; Fig. 2).

\section{Immunohistochemical Analysis of Eicosanoids in Meningiomas}

Immunohistochemical staining for COX-2 was performed in 124 human meningioma surgical specimens, with tonsil tissue used as a positive control. Ninety-five benign (77\%), 24 atypical (19\%), and five (4\%) anaplastic meningiomas stained positively for COX-2 (Table 1). The COX-2 immunoreactivity was found exclusively in the cytoplasm of all meningiomas studied. In fact $60(63 \%)$ of the 95 benign meningiomas, and 21 (88\%) of the 24 atypical meningiomas exhibited Grade 4 staining (Fig. 3D). Samples from 30 benign meningiomas $(32 \%)$ and three atypical tumors (12\%) displayed Grade 3 staining, and only five benign tumors (5\%) displayed Grade 2 staining. No Grade 0 or 1 stainings were noted. Normal cerebral cortex tissue stained for COX-2 displayed strong staining of the cytoplasm (Grade 4; Fig. 3C). Meningioma tissue samples were also stained for COX-1, 5-LO (Fig. 3F), and PTGER4. All 5-LO- and PTGER4-stained samples exhibited Grade 4 staining, whereas all corresponding COX-1 samples displayed Grade 2 staining. As a control, the antibodies to COX-2, 5-LO, and PTGER4 were treated with matching blocking peptides. The meningioma tissues showed no staining for these antibodies (Fig. 3E).

\section{Immunocytochemical Analysis of Eicosanoids in Meningiomas}

The immunocytochemical analysis for COX-1, COX-2, 5-LO, and PTGER4 was performed in the IOMM-Lee malignant meningioma cell line and in six meningiomas grown in cell culture. All seven specimens exhibited extensive cytoplasmic staining of COX-2, 5-LO, and PTGER4
(Grade 4; Fig. 4D-F), whereas cells stained with COX-1 displayed slightly weaker coloring (Grade 3; Fig. 4C). The COX-2, 5-LO, and PTGER4 antibodies were treated with matching blocking peptides. The benign meningioma samples showed no staining (Fig. 3E).

\section{Expression of Eicosanoid Protein in Meningiomas}

Twenty meningioma specimens, 84 meningioma primary cell cultures, the IOMM-Lee cell line, normal cerbral cortex and one surgical specimen of human dura were processed for Western blot experiments. Cytoplasmic and nuclear protein extracts were isolated. Both the COX-1 and -2 proteins have a molecular weight of approximately $70 \mathrm{kD}$. The molecular weight of 5-LO is approximately 78 $\mathrm{kD}$, and the loading control $\beta$-actin has a molecular weight of $42 \mathrm{kD}$. All meningiomas sampled exhibited staining of a large band of approximately $70 \mathrm{kD}$, and $78 \mathrm{kD}$ in the cytoplasm extracts (Fig. 5). None of the nuclear protein extracts showed staining for COX-1, COX-2, and 5-LO as expected by cytoplasmic proteins.

\section{Expression of Eicosanoid RNA in Meningiomas}

There was expression of eicosanoid RNA in 20 meningioma surgical specimens, 84 meningioma primary cell cultures, the IOMM-Lee cell line, normal cerebral cortex, and two dura surgical specimens, as shown on reverse-transcriptase PCR analysis. The PCR products of COX-1, COX-2, 5-LO, and PTGER4 were 143, 168, 207, and 167 $\mathrm{bp}$, respectively, and the product of the loading control ACTB was $183 \mathrm{bp}$. The semiquantitative derived mean value of COX-1 is significantly lower than the other investigated eicosanoids (compared with COX-2; $\mathrm{p}<0.01$, ttest). No change of expression in high-grade meningiomas was noted. The expression of COX-2 is comparable to normal cerebral parenchyma and dura in benign and atypical meningiomas. The higher expression of COX-2 in WHO

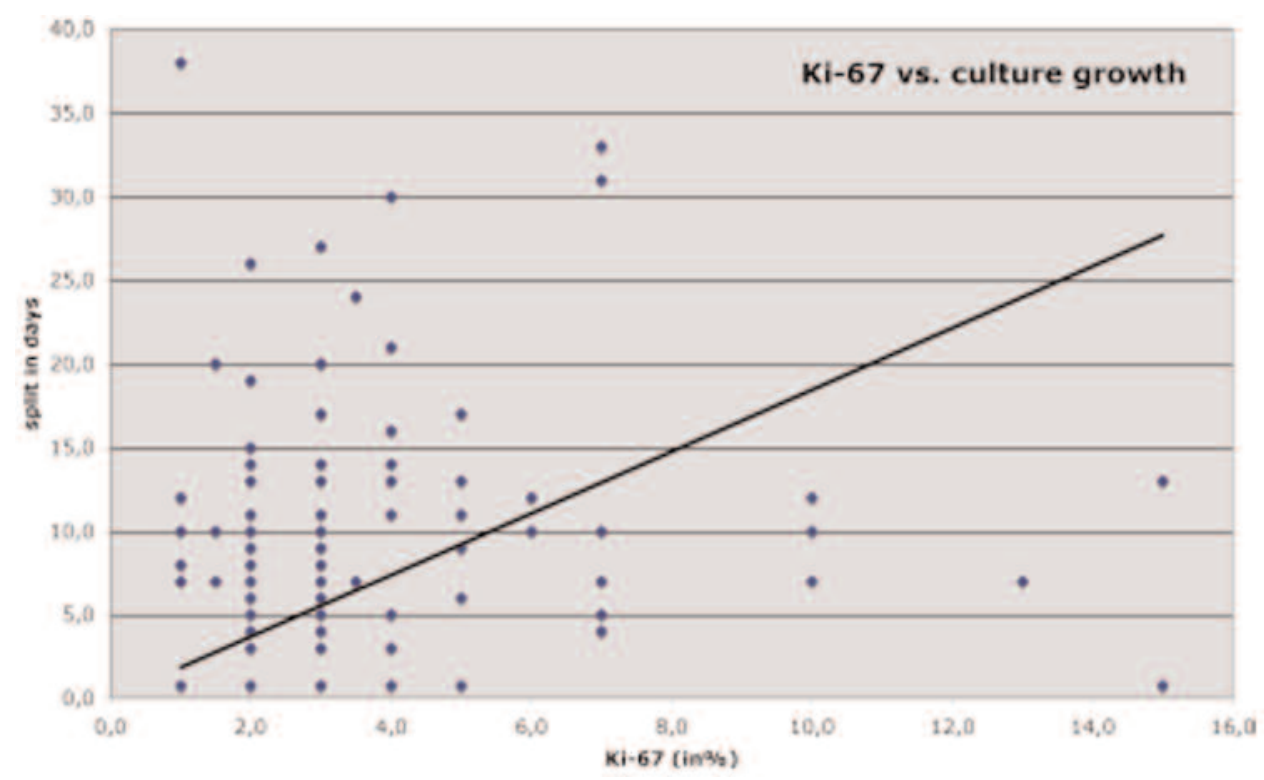

FIG. 2. Graph showing a linear regression of the Ki 67 proliferation index compared with culture growth. 


\section{TABLE 1}

Immunoreactivity to $C O X-2$ in 124 meningiomas stratified by $\mathrm{WHO}$ grade*

\begin{tabular}{lcccccc}
\hline \hline & \multicolumn{5}{c}{ Level of Cox-2 Reactivity } & \multirow{2}{*}{ Total } \\
\cline { 2 - 5 } WHO Grade & 0 & 1 & 2 & 3 & 4 & \\
\hline I (benign) & 0 & 0 & $5(4)$ & $30(24)$ & $60(48)$ & $95(77)$ \\
II (atypical) & 0 & 0 & 0 & $3(2)$ & $21(17)$ & $24(19)$ \\
III (anaplastic) & 0 & 0 & 0 & 0 & $5(4)$ & $5(4)$ \\
\hline
\end{tabular}

* No. of patients $(\%)$

Grade III meningiomas might be due to a bias based on few tissue samples. There are no significant differences in the expression of 5-LO in meningiomas and in normal cerebral cortex and dura. The mean value of PTGER4 is the same for normal cerebral cortex and dura as for WHO Grades I and II meningiomas (Fig. 6).

\section{Discussion}

Meningiomas are the most frequent benign tumors of the central nervous system. Although generally slow growing, these lesions continue to be a major cause of complications and death due to recurrence and unresponsiveness to adjuvant therapies. The finding that $\mathrm{AA}$ derivatives are expressed in meningiomas is fundamental and might create potential therapeutic opportunities..$^{6,14,37,44}$ Few reports exist on the constitutive expression of these enzymes in healthy brain tissue, as this should be the baseline for tumor investigation. ${ }^{18}$ In the central nervous system, COX-2 is expressed in components such as neurons, glia, and cerebro- vascular elements, ${ }^{2,19,28}$ or it can be induced by physiological or pathological stimuli. ${ }^{52}$ In accordance with the findings of Castelli and coworkers, ${ }^{6}$ who described similar expression of AA and metabolites in nine meningiomas and 10 healthy brain tissue specimens, we also found a high expression of AA derivatives in the healthy brain and in meningiomas of all grades. Ragel et al. ${ }^{44}$ described an almost ubiquitous (95\%) expression of COX-2 in 128 positively tested meningioma specimens. Because these researchers failed to compare their results with those from normal brain tissue, we validate their results adding data for healthy dura, normal brain tissue, and a substantial number of meningiomas of all different grades. Additionally, we verified the immunohistochemical findings through PCR, Western blot analysis, and immunocytochemistry, which should change the interpretation of previous reports proposing an upregulation of AA derivatives in meningiomas. ${ }^{37}$ Although the investigated enzymes are not upregulated in meningiomas compared with normal brain and dura, future chemotherapeutic strategies could still have numerous starting points in attacking key molecules in tumor development.

Arachidonic acid metabolism via the COX and the LO pathways leads to the production of a number of metabolites that may modulate many of the mechanisms involved in tumor initiation, growth, and dissemination. Nathoo and associates $^{38}$ have demonstrated 5-LO expression solely in the cytoplasm of neurons in the healthy adult brain, whereas, in most astrocytoma surgical specimens, strong staining for 5-LO was seen in the nuclei of tumor cells. The authors argued that the activation of tumor cells may lead to the translocation of 5-LO to the nuclear membrane rather than the plasma membrane; furthermore, the nuclear import
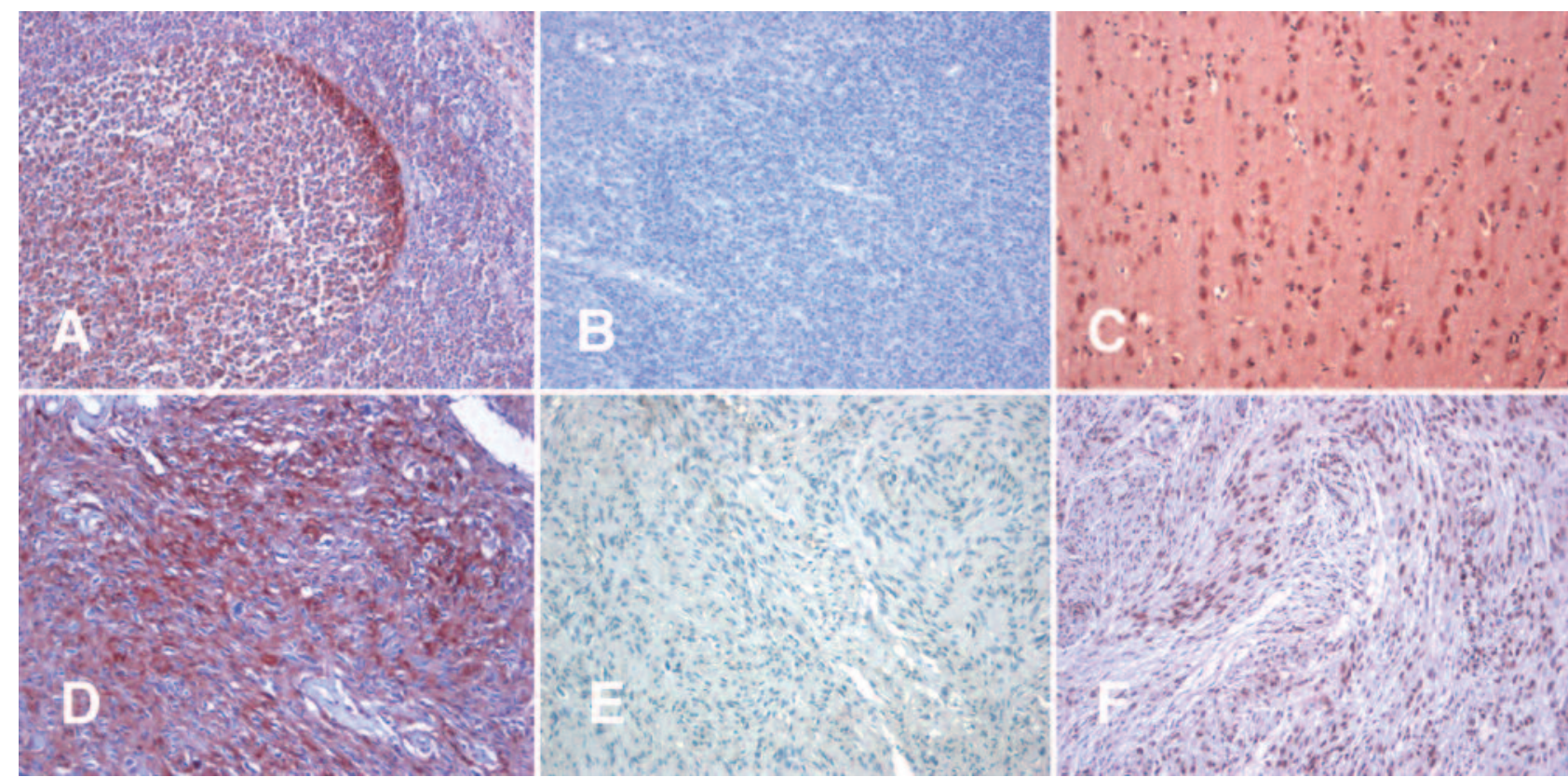

FIG. 3. Photomicrographs demonstrating eicosanoid expression. Positive immunoreactivity appears red. A: Positive control (human tonsil tissue) with strong COX-2 immunoreactivity in the cytoplasm. B: Negative control (tonsil tissue). C: Normal cerebral cortex showing strong COX-2 reactivity in neurons. D-F: Benign meningioma tissue. Strong COX-2 immunoreactivity is noted diffusely throughout the cytoplasm (D), no staining after incubation with COX2 antibody and COX-2 blocking peptide (E), and strong immunoreactivity to 5-LO is noted diffusely throughout the cytoplasm (F). NovaRED, original magnification $\times 10$. 

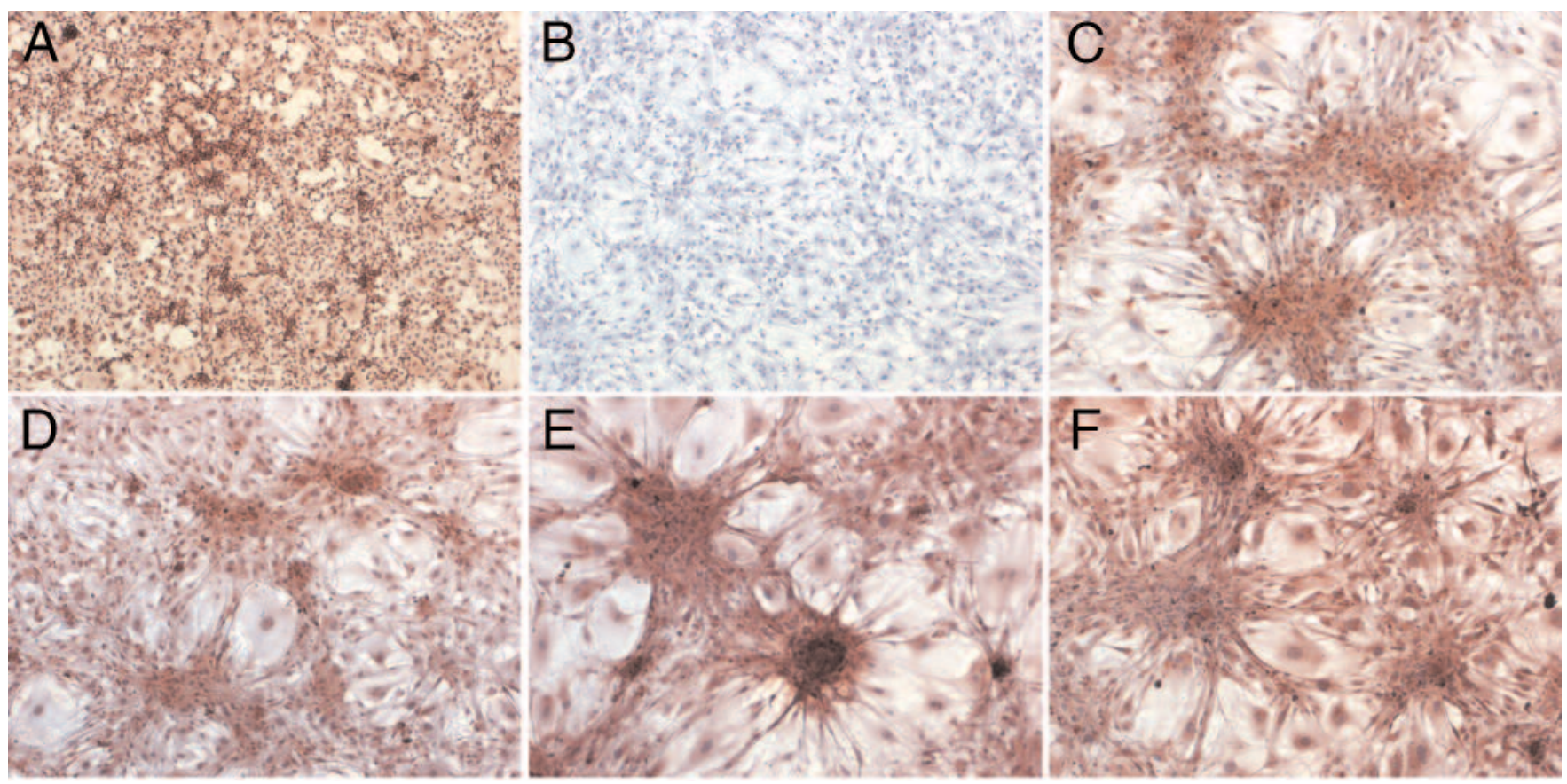

FIG. 4. Photomicrographs demonstrating expression of COX-2 in a culture of meningioma primary cells. Positive immunoreactivity appears red. A: Strong COX-2 immunoreactivity is seen in the cytoplasm of the IOMM-Lee cell line, used as the positive control. B: Negative control (meningioma tissue without primary antibody treatment). C: Grade 3 immunoreactivity to COX-1 is shown. D-F: Grade 4 COX-2 (D), 5-LO (E), and PTGER4 (F) immunoreactivity is noted diffusely throughout the cytoplasm in meningioma. NovaRED, original magnification $\times 4$.

sequence of 5-LO protein has been identified in certain leukocytes. ${ }^{22}$ We found high cytoplasmic expression of 5LO in almost all meningioma samples, with no expression in the nuclei of the tumor cells - either on immunohistochemical slides or on protein analysis. It remains to be determined whether 5-LO protein in meningiomas is functionally active and whether tumor cells express leukotriene receptors that could mediate cellular responses to 5-LO eicosanoids. Because 5-LO inhibitors are cytotoxic to cancer cell lines derived from several types of malignant tumors, further research into the potential use of 5-LO inhibitors for cancer prevention and treatment is warranted. ${ }^{1,15,16,38}$

Staining for COX-1 had a significantly lower expression than for the other observed eicosanoids, in agreement with the results of the reverse-transcriptase PCR analysis. These observations correlate with the findings of Matsuo et al., ${ }^{32}$ who reported a weak expression of COX-1 compared to COX-2 in normal human brain tissues, meningiomas, and other brain tumors. This could be due to the constitutive expression of COX-2 in the normal brain and particularly in meningiomas, leading to a takeover of functions elsewhere assigned to COX-1. Pai and colleagues ${ }^{41}$ reported that in addition to acting on its own receptor, $\mathrm{PGE}_{2}$ could also activate the epidermal growth factor receptor, providing another potential mechanism for the tumor promotion effect of COX enzymes. Their results not only further support the role of COX-2 as a tumor promoter in the intestine, but also point to $\mathrm{PGE}_{2}$ as the key mediator of the COX2-related susceptibility to colon cancer. Wu and cowork$e^{51}{ }^{51}$ published a study demonstrating inhibition of prolifer- ation and induction of apoptosis by celecoxib in human cholangiocarcinoma cells. Their results suggest that COX2 plays a central role in the production of $\mathrm{PGE}_{2}$ and that therefore specific inhibition with celecoxib, as a chemopreventive and chemotherapeutic agent of COX-2, inhibits proliferation and induces apoptosis of cells via suppression of $\mathrm{PGE}_{2}$ production. This study for the first time demonstrated that the brain intrinsic prostaglandin receptor PTGRE4 is strongly expressed in all meningioma specimens, pointing towards an important role of this enzyme in the development of meningiomas. Whether the compatible ligand of the receptor acts as a growth promotor or itself leads to molecular changes in the genetic program of the meningioma has to be elucidated.

As previously stated, COX-2 was expressed in all meningioma tissue samples. Because COX-2-derived metabolites may increase resistance to apoptosis, promote angiogenesis, and impair immune system surveillance, early prophylactic intervention with a COX-2 inhibitor may decrease tumorigenic progression in patients with malignant or invasive neoplasms. The tendency toward overexpression of COX-2 in more aggressive phenotypes of meningiomas suggests that COX-2 may play an important functional role in the later stages of invasive disease. Another hypothesis about increased COX-2 expression in more malignant meningiomas is that COX-2 levels could be an indicator of ischemia, ${ }^{3}$ supported by findings of necrotic areas within the tumor. Moreover, establishing a correlation between COX-2 expression and tumor recurrence or the Ki 67 proliferation index was not feasible, due to the overall high expression of COX-2 in meningiomas of 


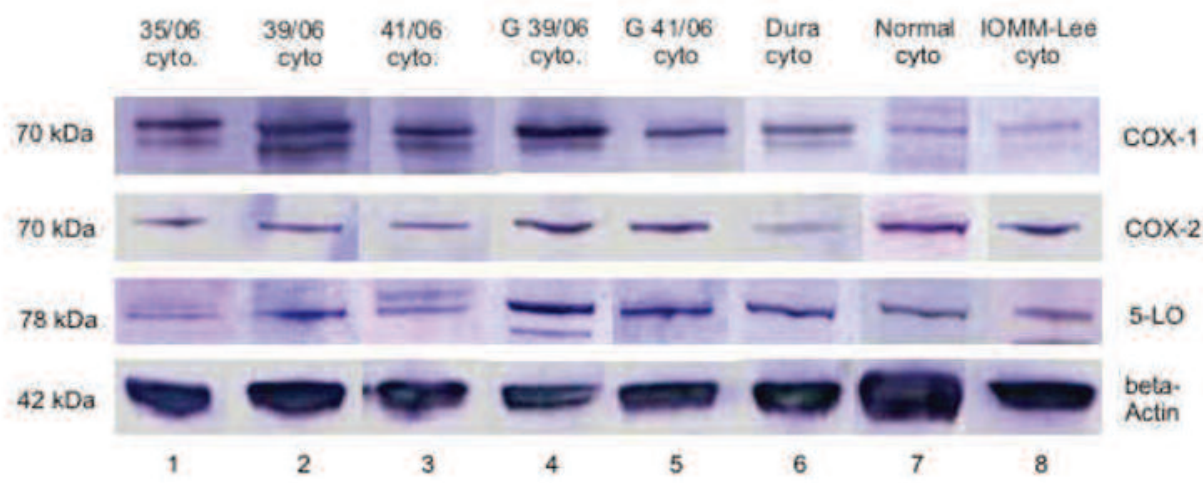

Fig. 5. Western blot analysis of three meningioma primary cells cultures, two meningioma surgical specimens, human dura, normal cerebral cortex, and IOMM-Lee cell line (numbered 1-8). Nuclear and cytoplasmic (cyto) protein extracts were isolated and probed with COX-1, COX-2, 5-LO, and beta-actin antibody (loading control). All nuclear protein extracts showed no staining, as expected by cytopasmic proteins. Lanes 1-5: All meningioma primary cell cultures and the fresh surgical specimens exhibited strong staining for COX-1, COX-2, and 5-LO. Lane 6: Human dura tissue stained positively for COX-1, COX-2 and 5-LO, although the band of COX-2 was weaker. Lane 7: Normal human cerebral cortex tissue showing corresponding staining for COX-2 and 5-LO, and weaker staining for COX-1. Lane 8: The positive control IOMM-Lee cell line exhibited strong staining for COX-2 and 5-LO, and weak staining for COX-1.

all grades. Based on the results of our study, it is not possible to declare COX-2 a prognostic indicator for tumor grading-as has been proposed by Lin et al. ${ }^{28}$ in meningiomas and Deininger and coworkers ${ }^{11}$ in oligodendrogliomasbecause no significant differences have been detected in eicosanoid expression among meningiomas of different WHO grades.

Radiation therapy has an important role in the management of recurrent meningiomas. In preclinical models of different cancers and clinical studies of gliomas, the addition of COX-2 inhibitors to radiotherapy can delay tumor regrowth compared with the use of either drugs or radiation alone. ${ }^{23,34,46,48}$ Pyo et al. ${ }^{42}$ found that in colorectal tumors expressing COX-2 that had been treated with radiation therapy and COX-2 inhibitors, there was a greater therapeutic response than to radiation therapy alone. Inhibition of COX-2 is augmented by the antiangiogenic effect of ionizing radiation through inhibition of nuclear factor- $\mathrm{\kappa B} .{ }^{4}$ The use of COX-2 inhibitors with ionizing radiation is a potentially attractive combination for recurrent meningioma.

In addition to the well-studied role of COX-2 in acute inflammatory processes, recent work clearly suggests that COX-2-derived metabolites contribute at multiple points in the early and late stages of carcinogenesis, including premalignant hyperproliferation, transformation, maintenance of tumor viability, growth, invasion, and metastatic spread. ${ }^{24}$ We confirm that COX-2 might be a key player in a number of biological pathways leading not only to cancer in general, but also to meningiomas. Current evidence indicates that COX-2 promotes tumor-specific angiogenesis, ${ }^{27,31}$ inhibits apoptosis, ${ }^{50}$ and induces proangiogenic factors such as vascular endothelial growth factor, ${ }^{29,47}$ inducible nitrogen oxide synthetase promoter, ${ }^{33}$ and interleukin- $6 .{ }^{54}$ Taken together, the epidemiological data and preclinical studies in animal models have generated compelling interest in the potential use of COX-2 inhibitors in the prevention and chemotherapy of human tumors. Clinical trials are

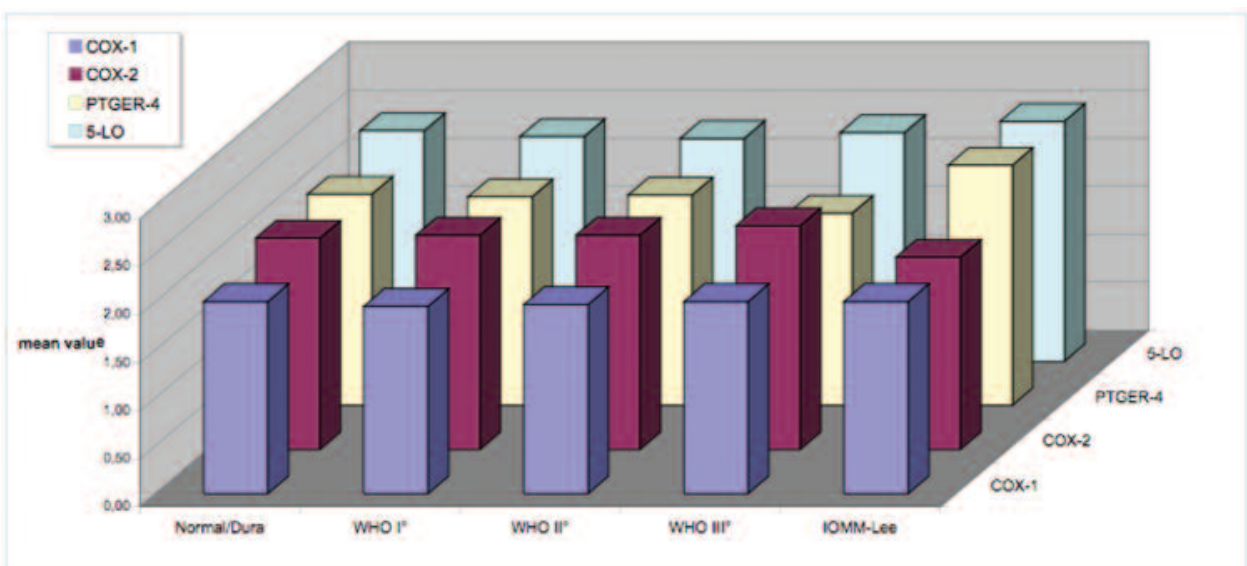

FIG. 6. Results of a PCR analysis of COX-1, COX-2, PTGER4, and 5-LO in meningiomas, normal cerebral cortex, human dura tissue, and the IOMM-Lee cell line. The mean values were determined by semiquantitative analysis. Betaactin was used to confirm equal loading among samples. 
necessary to determine whether COX-2 inhibitors will provide clinical benefit, as well as to define the intervention points during tumor progression that will allow for optimal efficacy.

\section{Conclusions}

The promising effect of COX-2 inhibitors in anaplastic meningiomas were recently reported by Ragel and colleagues ${ }^{43}$ although the effective dosage was far higher than the recommended plasma levels using standard dosages in humans. The association of COX-2 and meningioma is unique and represents a potential area for therapeutic intervention with selective COX-2 inhibitors, either as an adjunct to or in combination with radiation therapy. With the results of this study we confirm the existence of multiple attacking points in the eicosanoid cascade for a powerful chemotherapeutic treatment in recurrent meningiomas. Further studies are on the way to understand the influence and importance of single factors in this enzyme network so that treatment options can be explored.

\section{References}

1. Avis IM, Jett M, Boyle T, Vos MD, Moody T, Treston AM, et al: Growth control of lung cancer by interruption of 5-lipoxygenasemediated growth factor signaling. J Clin Invest 97:806-813, 1996

2. Bazan NG: COX-2 as a multifunctional neuronal modulator. Nat Med 7:414-415, 2001

3. Boado RJ, Pardridge WM, Vinters HV, Black KL: Differential expression of arachidonate 5-lipoxygenase transcripts in human brain tumors: evidence for the expression of a multitranscript family. Proc Natl Acad Sci U S A 89:9044-9048, 1992

4. Bradbury CM, Markovina S, Wei SJ, Rene LM, Zoberi I, Horikoshi N, et al: Indomethacin-induced radiosensitization and inhibition of ionizing radiation-induced NF-kappaB activation in HeLa cells occur via a mechanism involving p38 MAP kinase. Cancer Res 61:7689-7696, 2001

5. Buchanan FG, Wang D, Bargiacchi F, DuBois RN: Prostaglandin E2 regulates cell migration via the intracellular activation of the epidermal growth factor receptor. J Biol Chem 278: 35451-35457, 2003

6. Castelli MG, Butti G, Chiabrando C, Cozzi E, Fanelli R, Gaetani $\mathrm{P}$, et al: Arachidonic acid metabolic profiles in human meningiomas and gliomas. J Neurooncol 5:369-375, 1987

7. Chamberlain MC, Tsao-Wei DD, Groshen S: Salvage chemotherapy with CPT-11 for recurrent meningioma. J Neurooncol 78: 271-276, 2006

8. Cherukuri DP, Nelson MA: Glioma growth inhibition by selective COX-2 inhibitors via cyclooxygenase independent pathways: implications for therapy. Cancer Biol Ther 3:63-64, 2004

9. Cooper C, Jones HG, Weller RO, Walker V: Production of prostaglandins and thromboxane by isolated cells from intracranial tumors. J Neurol Neurosurg Psychiatry 47:579-584, 1984

10. Cui X, Yang SC, Sharma S, Heuze-Vourc'h N, Dubinett SM: IL4 regulates COX-2 and PGE2 production in human non-small cell lung cancer. Biochem Biophys Res Commun 343:995-1001, 2006

11. Deininger MH, Meyermann R, Trautmann K, Morgalla M, Duffner F, Grote EH, et al: Cyclooxygenase (COX)-1 expressing macrophages/microglial cells and COX-2 expressing astrocytes accumulate during oligodendroglioma progression. Brain Res 885:111-116, 2000

12. Deininger MH, Weller M, Streffer J, Mittelbronn M, Meyermann $\mathrm{R}$ : Patterns of cyclooxygenase- 1 and -2 expression in human gliomas in vivo. Acta Neuropathol 98:240-244, 1999
13. Fournier DB, Gordon GB: COX-2 and colon cancer: Potential targets for chemoprevention. J Cell Biochem Suppl 77:97-102, 2000

14. Gaetani P, Butti G, Chiabrando C, Danova M, Castelli MG, Riccardi A, et al: A study on the biological behavior of human brain tumors. Part I. Arachidonic acid metabolism and DNA content. J Neurooncol 10:233-240, 1991

15. Gupta S, Crofford LJ: An update on specific COX-2 inhibitors: the COXIBs. Bull Rheum Dis 50:1-4, 2001

16. Hennig R, Ding XZ, Tong WG, Schneider MB, Standop J, Friess $\mathrm{H}$, et al: 5-lipoxygenase and leukotriene $\mathrm{B}(4)$ receptor are expressed in human pancreatic cancers but not in pancreatic ducts in normal tissue. Am J Pathol 161:421-428, 2002

17. Hennig R, Grippo P, Ding XZ, Rao SM, Buchler MW, Friess H, et al: 5-lipoxygenase, a marker for early pancreatic intraepithelial neoplastic lesions. Cancer Res 65:6011-6016, 2005

18. Hétu PO, Riendeau D: Cyclo-oxygenase-2 contributes to constitutive prostanoid production in rat kidney and brain. Biochem $\mathbf{J}$ 391:561-566, 2005

19. Hoffmann C: COX-2 in brain and spinal cord implications for therapeutic use. Curr Med Chem 7:1113-1120, 2000

20. Jääskeläinen J: Seemingly complete removal of histologically benign intracranial meningioma: late recurrence rate and factors predicting recurrence in 657 patients. A multivariate analysis. Surg Neurol 26:461-469, 1986

21. Jiang WG, Douglas-Jones AG, Mansel RE: Aberrant expression of 5-lipoxygenase-activating protein (5-1OXAP) has prognostic and survival significance in patients with breast cancer. Prostaglandins Leukot Essent Fatty Acids 74:125-134, 2006

22. Jones SM, Luo M, Peters-Golden M, Brock TG: Identification of two novel nuclear import sequences on the 5-lipoxygenase protein. J Biol Chem 278:10257-10263, 2003

23. Kishi K, Petersen S, Petersen C, Hunter N, Mason K, Masferrer $\mathrm{JL}$, et al: Preferential enhancement of tumor radioresponse by a cyclooxygenase-2 inhibitor. Cancer Res 60:1326-1331, 2000

24. Koki AT, Masferrer JL: Celecoxib: a specific COX-2 inhibitor with anticancer properties. Cancer Control 9 (2 Suppl):28-35, 2002

25. Kokoglu E, Tuter Y, Sandikci KS, Yazici Z, Ulakoglu EZ, Sonmez H, et al: Prostaglandin E2 levels in human brain tumor tissues and arachidonic acid levels in the plasma membrane of human brain tumors. Cancer Lett 132:17-21, 1998

26. Kyritsis AP: Chemotherapy for meningiomas. J Neurooncol 29: 269-272, 1996

27. Leahy KM, Koki AT, Masferrer JL: Role of cyclooxygenases in angiogenesis. Curr Med Chem 7:1163-1170, 2000

28. Lin CC, Kenyon L, Hyslop T, Hammond E, Andrews DW, Curran WJ Jr, et al: Cyclooxygenase-2 (COX-2) expression in human meningioma as a function of tumor grade. Am J Clin Oncol 26: S98-S102, 2003

29. Liu Y, Cox SR, Morita T, Kourembanas S: Hypoxia regulates vascular endothelial growth factor gene expression in endothelial cells. Identification of a 5' enhancer. Circ Res 77:638-643, 1995

30. Marnett LJ, DuBois RN: COX-2: a target for colon cancer prevention. Annu Rev Pharmacol Toxicol 42:55-80, 2002

31. Masferrer JL, Leahy KM, Koki AT, Zweifel BS, Settle SL, Woerner BM, et al: Antiangiogenic and antitumor activities of cyclooxygenase-2 inhibitors. Cancer Res 60:1306-1311, 2000

32. Matsuo M, Yonemitsu N, Zaitsu M, Ishii K, Hamasaki Y, Fukuyama $\mathrm{K}$, et al: Expression of prostaglandin $\mathrm{H}$ synthase- 2 in human brain tumors. Acta Neuropathol 102:181-187, 2001

33. Melillo G, Musso T, Sica A, Taylor LS, Cox GW, Varesio L: A hypoxia-responsive element mediates a novel pathway of activation of the inducible nitric oxide synthase promoter. J Exp Med 182:1683-1693, 1995

34. Milas L, Kishi K, Hunter N, Mason K, Masferrer JL, Tofilon PJ: Enhancement of tumor response to gamma-radiation by an inhibitor of cyclooxygenase-2 enzyme. J Natl Cancer Inst 91: 1501-1504, 1999 
35. Mirimanoff RO, Dosoretz DE, Linggood RM, Ojemann RG, Martuza RL: Meningioma: analysis of recurrence and progression following neurosurgical resection. J Neurosurg 62:18-24, 1985

36. Nakasu S, Nakasu Y, Matsuda M: Meningioma recurrence. J Neurosurg 92:897-899, 2000

37. Nathoo N, Barnett GH, Golubic M: The eicosanoid cascade: possible role in gliomas and meningiomas. J Clin Pathol 57:6-13, 2004

38. Nathoo N, Prayson RA, Bondar J, Vargo L, Arrigain S, Mascha EJ, et al: Increased expression of 5-lipoxygenase in high-grade astrocytomas. Neurosurgery 58:347-354, 2006

39. Newton HB, Slivka MA, Stevens C: Hydroxyurea chemotherapy for unresectable or residual meningioma. J Neurooncol 49: 165-170, 2001

40. Ota S, Bamba H, Kato A, Kawamoto C, Yoshida Y, Fujiwara K: Review article: COX-2, prostanoids and colon cancer. Aliment Pharmacol Ther 16 (2 Suppl): 102-106, 2002

41. Pai R, Soreghan B, Szabo IL, Pavelka M, Baatar D, Tarnawski AS: Prostaglandin E2 transactivates EGF receptor: a novel mechanism for promoting colon cancer growth and gastrointestinal hypertrophy. Nat Med 8:289-293, 2002

42. Pyo H, Choy H, Amorino GP, Kim JS, Cao Q, Hercules SK, et al: A selective cyclooxygenase-2 inhibitor, NS-398, enhances the effect of radiation in vitro and in vivo preferentially on the cells that express cyclooxygenase-2. Clin Cancer Res 7:2998-3005, 2001

43. Ragel BT, Jensen RL, Gillespie DL, Prescott SM, Couldwell WT: Celecoxib inhibits meningioma tumor growth in a mouse xenograft model. Cancer 109:588-597, 2007

44. Ragel BT, Jensen RL, Gillespie DL, Prescott SM, Couldwell WT: Ubiquitous expression of cyclooxygenase-2 in meningiomas and decrease in cell growth following in vitro treatment with the inhibitor celecoxib: potential therapeutic application. J Neurosurg 103:508-517, 2005

45. Schrell UM, Rittig MG, Anders M, Koch UH, Marschalek R, Kiesewetter F, et al: Hydroxyurea for treatment of unresectable and recurrent meningiomas. II. Decrease in the size of meningiomas in patients treated with hydroxyurea. J Neurosurg 86: 840-844, 1997
46. Shin YK, Park JS, Kim HS, Jun HJ, Kim GE, Suh CO, et al: Radiosensitivity enhancement by celecoxib, a cyclooxygenase (COX)-2 selective inhibitor, via COX-2-dependent cell cycle regulation on human cancer cells expressing differential COX-2 levels. Cancer Res 65:9501-9509, 2005

47. Shweiki D, Itin A, Soffer D, Keshet E: Vascular endothelial growth factor induced by hypoxia may mediate hypoxia-initiated angiogenesis. Nature 359:843-845, 1992

48. Sminia P, Kuipers G, Geldof A, Lafleur V, Slotman B: COX-2 inhibitors act as radiosensitizer in tumor treatment. Biomed Pharmacother 59 (2 Suppl): S272-S275, 2005

49. Soumaoro LT, Iida S, Uetake H, Ishiguro M, Takagi Y, Higuchi $\mathrm{T}$, et al: Expression of 5-lipoxygenase in human colorectal cancer. World J Gastroenterol 12:6355-6360, 2006

50. Tsujii M, DuBois RN: Alterations in cellular adhesion and apoptosis in epithelial cells overexpressing prostaglandin endoperoxide synthase 2. Cell 83:493-501, 1995

51. Wu AW, Gu J, Ji JF, Li ZF, Xu GW: Role of COX-2 in carcinogenesis of colorectal cancer and its relationship with tumor biological characteristics and patients' prognosis. World J Gastroenterol 9:1990-1994, 2003

52. Yamagata K, Andreasson KI, Kaufmann WE, Barnes CA, Worley PF: Expression of a mitogen-inducible cyclooxygenase in brain neurons: regulation by synaptic activity and glucocorticoids. Neuron 11:371-386, 1993

53. Yamasaki F, Yoshioka H, Hama S, Sugiyama K, Arita K, Kurisu K: Recurrence of meningiomas. Cancer 89:1102-1110, 2000

54. Yan SF, Tritto I, Pinsky D, Liao H, Huang J, Fuller G, et al: Induction of interleukin 6 (IL-6) by hypoxia in vascular cells. Central role of the binding site for nuclear factor-IL-6. J Biol Chem 270:11463-11471, 1995

Manuscript submitted July 13, 2007

Accepted September 9, 2007.

Address correspondence to: Florian Roser, M.D., Ph.D., Department of Neurosurgery, University of Tübingen, Hoppe-SeylerStr. 3, 72076 Tübingen, Germany. e-mail: f.roser@gmx.de. 